PPPL $-256 \mathrm{C}$

DE89 00462.7

\title{
Approximation for Nonresonant Beam Target Fusion Reactivities
}

\author{
D. R. Mikkelsen \\ Princeton Plasma Physics Laboratory \\ P.O. Box 451 \\ Princeton, NJ 08543
}

\begin{abstract}
A bstract
The beam target fusion reactivity for a monoenergetic beam in a Maxwellian target is approximately evaluated for nonresonant reactions. The approximation is accurate for the DD and TT fusion reactions to better than $4 \%$ for all beam energies up to $300 \mathrm{keV}$ and all ion temperatures up to $2 / 3$ of the beam energy.
\end{abstract}




\section{Introduction}

A series of recent experiments has led to improved determinations of the cross sections for the DT $[1,2], \mathrm{DD}[3-5], \mathrm{DHe}^{3}[4,6]$ (see discussion in $7)$, and TT 3 i fusion reactions. The present work exploits the simple energy dependence of nonresonant reaction cross sections to calculate the beam target reactivity. The method is parallel to that used by Bahcall[ $[8 j$ to calculate the thermonuclear reactivity.

A similar method has been used by Core[9] to develop formulae for the DT reaction. Linfortunately, the resonance in that reaction at a center of mass energ: of $50 \mathrm{keV}$ ) complicates the energy dependence of the cross section. As a result, the Bahcall and Core expressions for the thermonuclear reactivity are inaccurate for ion temperatures above $\sim 15 \mathrm{keV}$ (at $30 \mathrm{keV}$ the approximations are $50 \%$ too large). Core's beam target reactivity is also accurate only for relatively low values of the ion temperature. While simple fits to the numerically calculated thermonuclear reactivity of the resonant DT and $\mathrm{DHe}^{3}$ reactions exist[10], there are apparent]y no similar fits to the beam target reactivity which are valid for the full range of beam voltages and target temperatures of interest.

The absence of a resonance in the DD and TT reactions permits an accurate, simple evaluation of the thermonuclear[5] and beam target reactivities (below).

\section{Approximate beam target reactivity}

The beam target reactivity for a monoenergetic beam at a speed $v_{b}=$ $\sqrt{2 E_{\mathrm{b}} / m_{\mathrm{b}}}$ reacting with a Maxwellian target with a thermal speed $\sigma_{\mathrm{th}}=$ $\sqrt{2 T_{\mathrm{i}} / m_{\mathrm{i}}}$ is $[11]$

$$
(\sigma v)_{b \mathrm{~b}}=\frac{1}{v_{b} \sigma_{\mathrm{th}} \sqrt{\pi}} \int_{0}^{\infty} \sigma v^{2}\left[e^{-\left(\frac{v-v_{h}}{\sigma_{h h}}\right)^{2}}-e^{-\left(\frac{v+v_{\mathrm{b}}}{\sigma_{h}}\right)^{2}}\right] d v
$$

The complete beam target reactivity is obtained by averaging $(\sigma v)_{b}$ over the beam nnergy distribution. 
The evaluation of the integral can be simplified by defining the 'astrophysical S function' as the slowly rarying part of the cross section:

$$
\sigma\left(E_{\mathrm{cm}}\right) \equiv S\left(E_{\mathrm{cm}}\right) /\left[E_{\mathrm{cm}} \exp \left(-b / \sqrt{E_{\mathrm{cm}}}\right)\right]
$$

where $b=\pi a Z_{b} Z_{t} \sqrt{2 \mu c^{2}}=31.4 \sqrt{2 \mu / m_{\mathrm{D}}} \sqrt{\mathrm{keV}}, E_{\mathrm{rm}}=\mu v^{2} / 2$, and $\mu=$ $m_{\mathrm{b}} m_{\mathrm{t}} /\left(m_{\mathrm{b}}+m_{\mathrm{t}}\right)$ is the reduced mass.

The second exponential in Eq. (1) is ignored below because direct calculation shows that it makes a negligible contribution in all cases of interest. i.e., $E_{b} \leq 1 \mathrm{MeV}$ and $T_{i} \leq 2 E / 3$. This can be easily understood by noting that since $\sigma\left(E_{\mathrm{cm}}\right)$ is a strongly rising function of energy, the complete integrand is maximized at a speed of $v>v_{b}$, so $\left(\left(v+v_{b}\right) / \sigma_{t h}\right)^{2}>4\left(v_{b} / \sigma_{t h}\right)^{2}$, and if $E_{\mathrm{b}} \geq 3 T_{i} / 2$ the second exponential is of order $e^{-6}$ in the region where the first exponential is of order 1.

Using Eq.(2) we rewrite the dominant term of Eq.(1) as

$$
(\sigma v)_{b t}=\frac{2}{\mu v_{b} \sigma_{\mathrm{th}} \sqrt{\pi}} \int_{0}^{\infty} S\left(E_{\mathrm{cm}}\right) \exp \left[-\left(\frac{B}{v}+\left(\frac{v-v_{\mathrm{b}}}{\sigma_{\mathrm{th}}}\right)^{2}\right)\right] d v,
$$

where $B=2 \pi a Z_{b} Z_{t} c$. The rapid energv dependence of the integrand is isolated in the exponential, the argument of which

$$
F(v)=B / v+\left(\frac{v-v_{b}}{\sigma_{\imath h}}\right)^{2}
$$

is maximized at a speed $v_{*}$, where

$$
v_{*}^{2}\left(v_{c}-v_{b}\right)=\sigma_{t h}^{2} B / 2 \text {. }
$$

The solution is

$$
v_{\text {. }}=v_{\mathrm{b}}[1 / 3+\sqrt[3]{R+D}+\sqrt[3]{R-D}]
$$

where $R=\beta / 2+1 / 27, D=\sqrt{\beta(\beta / 4+1 / 27)}$, and $\beta=\left(\sigma_{\mathrm{th}} / \iota_{\mathrm{b}}\right)^{2} B / 2 v_{\mathrm{b}}$. Letting

$$
v=v_{\bullet}+\sigma_{\mathrm{th}} u
$$

and expanding $F(v)$ we have

$$
F(v)=F\left(v_{-}\right)+u^{2}\left[1+2\left(v_{-}-v_{\mathrm{b}}\right) /\left(v_{-}+\sigma_{\mathrm{th}} u\right)\right]
$$


Expanding $F(v)$ and $S\left(E_{\mathrm{cm}}\right)$ in a Taylor series in $u$ about $u$, (and neglecting higher order derivatives of $S$ ) we are led to

$$
\begin{aligned}
& (\pi v)_{b \mathrm{~b}} \sim \frac{2}{\mu v_{\mathrm{b}} v^{\prime} \pi} \int_{-v_{0} / \sigma_{\mathrm{hh}}}^{\infty} S\left(E_{-}\right)-S^{\prime}\left(E_{-}\right) \mu\left(v_{-} \sigma_{\mathrm{th}} u-\sigma_{\mathrm{th}}^{2} u^{2} / 2\right) \exp -F\left(v_{-}\right) \\
& x \exp \left(-\gamma u^{2}\right) 1-2\left(v_{0}-t_{\mathrm{b}}\right)\left(\sigma_{\mathrm{th}} t^{3}{ }_{i} v_{0}^{2}\right)\left(1-\sigma_{\mathrm{th}} u_{i} v_{0}\right) d u \text {. }
\end{aligned}
$$

where $E_{0}=\mu v_{0}^{2} \cdot 2 . \gamma=1-2\left(v_{*}-v_{\mathrm{b}}\right) / v_{*}$, and we have assumed $2\left(v_{0}-\right.$ $\left.\nu_{\mathrm{b}}\right) \sigma_{\mathrm{th}} u^{3}{ }^{2} \mathrm{v}^{2}<1$.

lery small additional errors are incurred by extending the lower integration limit to $-\infty$ and keeping only the terms of order $u^{0}, u^{2}$, and $u^{4}$ to give the final result

$$
\begin{aligned}
(\sigma v)_{\mathrm{t} t} \simeq & \sigma\left(E_{*}\right) v_{*} \frac{\exp \left[-\left(\frac{v_{+}-v_{\mathrm{b}}}{\sigma_{\mathrm{th}}}\right)^{2}\right]\left(v_{-} / v_{\mathrm{b}}\right)}{\sqrt{\gamma}}\left\{1+\frac{\mu \sigma_{\mathrm{th}}^{2} S^{\prime}\left(E_{-}\right)}{4 \gamma S\left(E_{-}\right)}\right. \\
& \left.+\frac{3\left(v_{-}-v_{\mathrm{b}}\right)}{2 v_{-} \gamma^{2}}\left[\frac{\mu \sigma_{\mathrm{th}}^{2} S^{\prime}\left(E_{-}\right)}{S\left(E_{-}\right)}-\left(\frac{\sigma_{\mathrm{th}}}{v_{*}}\right)^{2}\right]\right\}
\end{aligned}
$$

Althuugh Core's 9] beam target reactivity included the smaller exponential in Eq. (1) and did not extend the integral in Eq. (6) to $-\infty$. his result differs from the lowest order term in Eq. $(\vec{r})$ by much less than $1 \%$.

\section{Application to the DD and TT reactions}

The DD and TT fusion reaction cross sections are well approxinated by

$$
S\left(E_{\mathrm{cm}}\right)=S_{\mathrm{o}}+S_{1} E_{\mathrm{cm}},
$$

for $E_{\mathrm{b}} \leq E_{\max }$ where $\left(S_{o}, S_{1}, E_{\max }\right)$ are ( $53.55 \mathrm{keV}$ barn, $0.3282 \mathrm{barn}$, i00 leV) for the $\mathrm{D}(\mathrm{d}, \mathrm{n}) \mathrm{He}^{3}$ reaction $[5]$, ( $55.72 \mathrm{keV}$ barn, $\left.0.1868 \mathrm{barn}, 3 \mathrm{MeV}\right)$ for the $\mathrm{D}(\mathrm{d}, \mathrm{P}) \mathrm{T}$ reaction $|5|$, and $(190 \mathrm{keV}$ barn, $-0.2 \mathrm{barn}, 700 \mathrm{keV})$ for the TT reaction[3].

The new cross section measurements for the $\mathrm{D}(\mathrm{d}, \mathrm{n}) \mathrm{He}^{3}$ reaction are $5 \%$ lower than Duane's fit[12] for $100 \mathrm{keV}<E_{\mathrm{b}}<150 \mathrm{keV}$, and 10-15\% higher than Peres' fit [10].

A simple estimate of the importance of the target temperature is given by the lowest order correction to the cold target reactivity: $\sigma\left(E_{\mathrm{am}}=E_{\mathrm{b}} / 2\right) v_{\mathrm{b}}$. 
For the D(d.n)He reaction at $E_{\mathrm{b}}=120 \mathrm{kel}, \sigma \mathrm{r}-E^{\mathrm{l}} \mathrm{s} . E_{0}=E_{\mathrm{b}}(1-\beta)^{2}$. $3=2 T_{1}, E_{\mathrm{b}}$, and

$$
\frac{\exp \left[-\left\{\frac{v_{-}-v_{b}}{\sigma_{\mathrm{th}}}\right)^{2}\right]\left(v_{-}\left(v_{3}\right)\right.}{\sqrt{\gamma}} \simeq(1-2 \beta),
$$

so

$$
(\sigma v)_{b x}=\sigma\left(E_{\mathrm{b}} / 2\right) v_{\mathrm{b}}\left(l+3 T_{\mathrm{i}} / E_{\mathrm{b}}\right) .
$$

This shows that even when $T_{\mathrm{j}}=0.1 E_{\mathrm{b}}$ the finite temperature enhancement of $(\sigma v)_{b e}$ is significant. The enhancement [calculated from Eq. (1)] for a range of beam energies and temperatures is given in Table 1.

For both DD reactions the first two terms of Eq. (7) approximate $(\sigma v)_{b e}$ as ralculated numerically from $E q$. (1) to $4 \%$ for $E_{\mathrm{b}}<300 \mathrm{keV}$ and $T_{1}<2 E_{1},{ }^{\prime} 3$. The negative slope of $S\left(E_{\mathrm{cm}}\right)$ for the TT reaction leads to less rancellation in the third term of Eq. (7) and all terms are required to achieve a $t \%$ level of accuracy over this tange of beam energies and temperatures.

If the target temperature is restricted to be no higher than $30 \mathrm{keV}$, however, the first tri $\mathrm{M}$ of $\mathrm{Eq}$. ( $\mathrm{T}$ ) is accurate to $3 \%$ for all three of the reactions considered here over the entire range of $E_{\mathrm{b}}$ for which the cross sections fits are valid (see Fig. 1 for $E_{\mathrm{b}}<200 \mathrm{keV}$ ). In most circumstances, therefore, the lowest order approximation provides a convenient, computationally inexpensive evaluation of Eq. (1) for the DD and TT reactions.

\section{Acknowledgment}

It is a pleasure to thank P. Colestock, G. Hammett, and H. Towner for their comments on the manuseript. This work was supported by U.S. DoE Contract No. DE-AC02-76-CHO-3073. 


\section{References}

I. JARHE, N.. BROWX, K. E.. and HARDEKOPF. R. A., Phys. Res. 29 (1984) 2031.

2 BROWZ, R. E., JARMIE. A., and HALE. G. M., Phys. Kev. 35 (198i) 1999.

3 JARMIE, N. and BROLN, R. E., Rucl. Instrum. Methods B10/11 (1985) 405.

4. KRALSS, A.. BECKER. H. W., TRALTVETTER, H. P., and ROLFS, C.. Nucl. Phys. A465 (198i) 150.

5. BROWN, R. E. and JARMIE. Y., Differential ctoss sections for ${ }^{2} \mathrm{H}(\mathrm{d}, \mathrm{p})^{3} \mathrm{H}$ and ${ }^{2} \mathrm{H}(\mathrm{d}, \mathrm{n})^{3} \mathrm{He}$ at low energies, to be submitted to Phys. Rer. C.

6 MÖLlER, $W$. and BESSENBACHER, F, Nucl. Instrum. Methods 188 (1980) 111 .

־. JAR.IIE, $\times$., Requirements for charged-perticle reaction cross sections in the D-T. D-D, T-T, and D. ${ }^{3}$ He fuel cycles, Technical Report LA-LR86-3705, Los Alamos National Laboratory, 1986.

8. BAHCALL, J. N.. Astrophys. J. 143 (1966) 259.

9. CORE, W. G. F., A note on the computation of thermonuclear reactivities in plasma-fusion applications, Technical Report JET-IR(B7)11, Joint European Torus, 1987.

10; PERES, A., J. Appl. Phys. 50 (1979) 5569.

(11) MILEY, G. H., TOWNER, H., and IVICH, N., Fusion cross sections and reactivities, Technical Report COO-2218-17, University of Illinois, 1974 .

12! DUANE, B. H., The Pacific Northwest Laboratory annual report on controlled thermonuclear reactor technology, Technical Report BNWL1685, Batlelle Parific Northwest Laboratory, 1972. 


\begin{tabular}{|c|c|c|c|c|}
\hline \multirow{2}{*}{$\begin{array}{c}E_{\mathrm{b}} \\
\text { (kel) }\end{array}$} & \multirow{2}{*}{$\left(10^{-24} \mathrm{~m}^{3} \mathrm{ser}\right)$} & \multicolumn{3}{|c|}{ Enhancement: $(\sigma v)_{b t} \sigma v_{\mathrm{b}}$} \\
\hline & & $T_{1}=5 \mathrm{kel}$ & $T_{1}=15 \mathrm{kel}$ & $T_{1}=30 \mathrm{kel}$ \\
\hline 50 & 1.01 & 1.8 & $\overline{3.3}$ & 5.6 \\
\hline 100 & 5.11 & 1.2 & 1.6 & 2.1 \\
\hline 150 & 10.5 & 1.1 & 1.3 & 1.5 \\
\hline
\end{tabular}

\section{Figure caption}

Figure 1. The relative error for the first term of $5 q .(7)$ compared to numerical integration of Eq. (1). The relutive error of the $\mathrm{D}(\mathrm{d}, \mathrm{p}) \mathrm{T}$ reaction is less than $1 \%$ for all $T_{i}$ less than 30 kel. 
\#88P0120

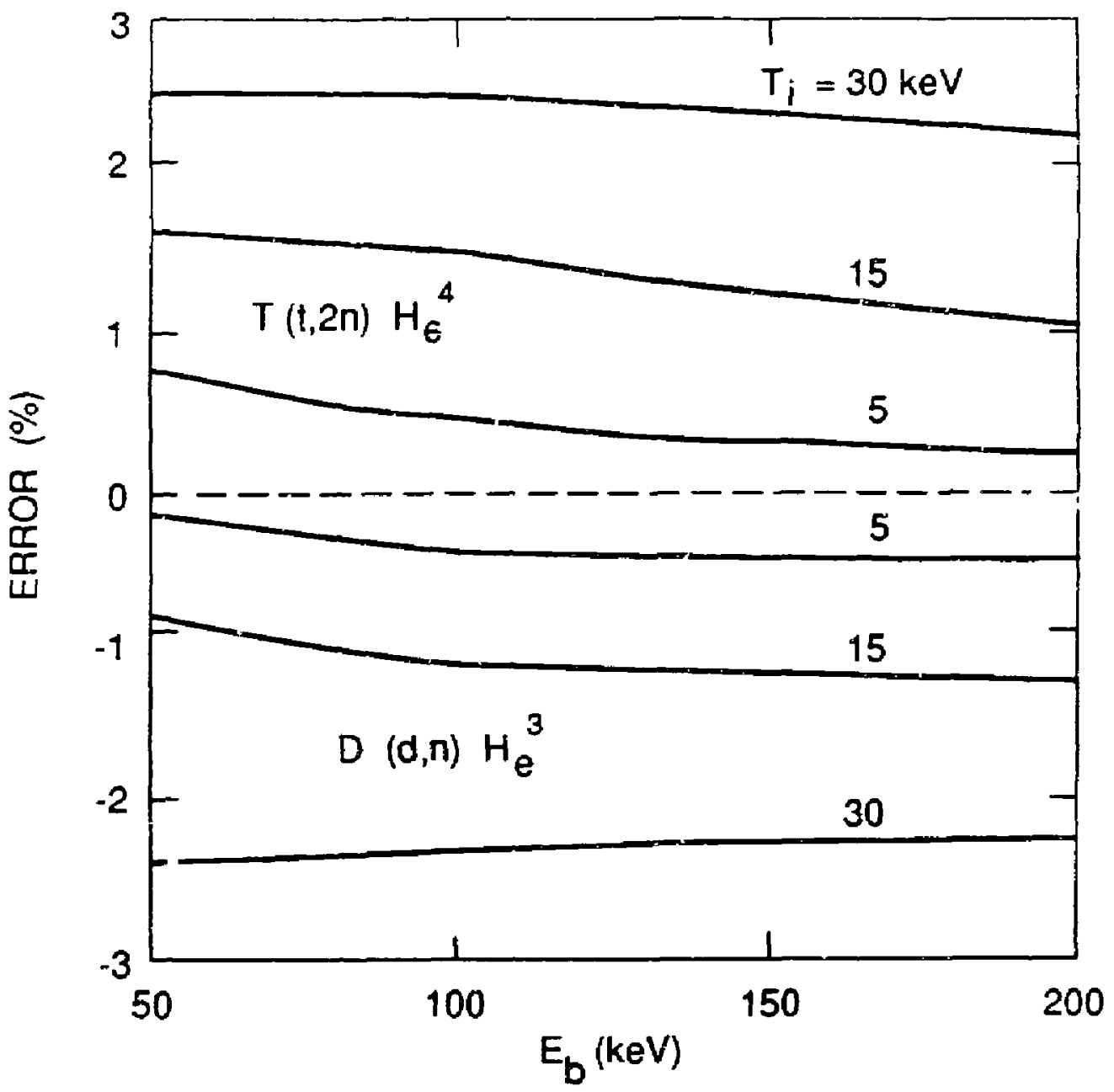

Fig. 1 